\title{
Identities of Symmetry for Euler Polynomials Arising from Quotients of Fermionic Integrals Invariant under $S_{3}$
}

\author{
Dae San Kim and Kyoung Ho Park \\ Department of Mathematics, Sogang University, Seoul 121-742, Republic of Korea \\ Correspondence should be addressed to Dae San Kim, dskim@sogang.ac.kr \\ Received 16 February 2010; Accepted 13 April 2010 \\ Academic Editor: Yeol J. E. Cho
}

Copyright (C) 2010 D. S. Kim and K. H. Park. This is an open access article distributed under the Creative Commons Attribution License, which permits unrestricted use, distribution, and reproduction in any medium, provided the original work is properly cited.

We derive eight basic identities of symmetry in three variables related to Euler polynomials and alternating power sums. These and most of their corollaries are new, since there have been results only about identities of symmetry in two variables. These abundances of symmetries shed new light even on the existing identities so as to yield some further interesting ones. The derivations of identities are based on the $p$-adic integral expression of the generating function for the Euler polynomials and the quotient of integrals that can be expressed as the exponential generating function for the alternating power sums.

\section{Introduction and Preliminaries}

Let $p$ be a fixed odd prime. Throughout this paper, $\mathbb{Z}_{p}, \mathbb{Q}_{p}, \mathbb{C}_{p}$ will, respectively, denote the ring of $p$-adic integers, the field of $p$-adic rational numbers, and the completion of the algebraic closure of $\mathbb{Q}_{p}$. For a continuous function $f: \mathbb{Z}_{p} \rightarrow \mathbb{C}_{p}$, the $p$-adic fermionic integral of $f$ is defined by

$$
\int_{\mathbb{Z}_{p}} f(z) d \mu_{-1}(z)=\lim _{N \rightarrow \infty} \sum_{j=0}^{p^{N}-1} f(j)(-1)^{j} .
$$

Then it is easy to see that

$$
\int_{\mathbb{Z}_{p}} f(z+1) d \mu_{-1}(z)+\int_{\mathbb{Z}_{p}} f(z) d \mu_{-1}(z)=2 f(0) .
$$


Let ||$_{p}$ be the normalized absolute value of $\mathbb{C}_{p}$, such that $|p|_{p}=1 / p$, and let

$$
E=\left\{\left.t \in \mathbb{C}_{p}|| t\right|_{p}<p^{-1 /(p-1)}\right\}
$$

Then, for each fixed $t \in E$, the function $f(z)=e^{z t}$ is analytic on $\mathbb{Z}_{p}$, and by applying (1.2) to this $f$, we get the $p$-adic integral expression of the generating function for Euler numbers $E_{n}$ :

$$
\int_{\mathbb{Z}_{p}} e^{z t} d \mu_{-1}(z)=\frac{2}{e^{t}+1}=\sum_{n=0}^{\infty} E_{n} \frac{t^{n}}{n !} \quad(t \in E)
$$

So we have the following $p$-adic integral expression of the generating function for the Euler polynomials $E_{n}(x)$ :

$$
\int_{\mathbb{Z}_{p}} e^{(x+z) t} d \mu_{-1}(z)=\frac{2}{e^{t}+1} e^{x t}=\sum_{n=0}^{\infty} E_{n}(x) \frac{t^{n}}{n !} \quad\left(t \in E, x \in \mathbb{Z}_{p}\right)
$$

Let $T_{k}(n)$, denote the alternating $k$ th power sum of the first $(n+1)$ nonnegative integers, namely,

$$
T_{k}(n)=\sum_{i=0}^{n}(-1)^{i} i^{k}=(-1)^{0} 0^{k}+(-1)^{1} 1^{k}+(-1)^{2} 2^{k}+\cdots+(-1)^{n} n^{k} .
$$

In particular,

$$
T_{0}(n)=\left\{\begin{array}{ll}
1, & \text { if } n \equiv 0(\bmod 2), \\
0, & \text { if } n \equiv 1(\bmod 2),
\end{array} \quad T_{k}(0)= \begin{cases}1, & \text { for } k=0 \\
0, & \text { for } k>0\end{cases}\right.
$$

From (1.4) and (1.6), one easily derives the following identities: for any odd positive integer $w$,

$$
\frac{\int_{\mathbb{Z}_{p}} e^{x t} d \mu_{-1}(x)}{\int_{\mathbb{Z}_{p}} e^{w y t} d \mu_{-1}(y)}=\sum_{i=0}^{w-1}(-1)^{i} e^{i t}=\sum_{k=0}^{\infty} T_{k}(w-1) \frac{t^{k}}{k !} \quad(t \in E) .
$$

In what follows, we will always assume that the $p$-adic fermionic integrals of the various exponential functions on $\mathbb{Z}_{p}$ are defined for $t \in E$ (cf., (1.3)), and therefore it will not be mentioned.

Many authors have done much work on identities of symmetry involving Bernoulli polynomials or Euler polynomials or $q$-Bernoulli polynomials or $q$-Euler polynomials. We let the reader refer to the papers in [1-20]. In connection with Bernoulli polynomials and power sums, these results were generalized in [21] to obtain identities of symmetry involving three variables in contrast to the previous works involving just two variables.

In this paper, we will produce 8 basic identities of symmetry in three variables $w_{1}, w_{2}$, $w_{3}$ related to Euler polynomials and alternating power sums (cf., (4.8), (4.9), (4.12), (4.16), 
(4.20), (4.23), (4.25), and (4.26)). These and most of their corollaries seem to be new, since there have been results only about identities of symmetry in two variables in the literature. These abundances of symmetries shed new light even on the existing identities. For instance, it has been known that (1.9) and (1.10) are equal and (1.11) and (1.12) are so (cf., [3, Theorems $5,7])$. In fact, (1.9)-(1.12) are all equal, as they can be derived from one and the same $p$ adic integral. Perhaps, this was neglected to mention in [3]. Also, we have a bunch of new identities in (1.13)-(1.16). All of these were obtained as corollaries (cf., Corollary 4.9, 4.12, 4.15 ) to some of the basic identities by specializing the variable $w_{3}$ as 1 . Those would not be unearthed if more symmetries had not been available.

Let $w_{1}, w_{2}$ be any odd positive integers. Then we have

$$
\begin{aligned}
\sum_{k=0}^{n}\left(\begin{array}{l}
n \\
k
\end{array}\right) E_{k}\left(w_{1} y_{1}\right) T_{n-k}\left(w_{2}-1\right) w_{1}^{n-k} w_{2}^{k} \\
=\sum_{k=0}^{n}\left(\begin{array}{l}
n \\
k
\end{array}\right) E_{k}\left(w_{2} y_{1}\right) T_{n-k}\left(w_{1}-1\right) w_{2}^{n-k} w_{1}^{k} \\
=w_{1}^{n} \sum_{i=0}^{w_{1}-1}(-1)^{i} E_{n}\left(w_{2} y_{1}+\frac{w_{2}}{w_{1}} i\right) \\
=w_{2}^{n} \sum_{i=0}^{w_{2}-1}(-1)^{i} E_{n}\left(w_{1} y_{1}+\frac{w_{1}}{w_{2}} i\right) \\
=\sum_{k+l+m=n}\left(\begin{array}{c}
n \\
k, l, m
\end{array}\right) E_{k}\left(y_{1}\right) T_{l}\left(w_{1}-1\right) T_{m}\left(w_{2}-1\right) w_{1}^{k+m} w_{2}^{k+l} \\
=w_{1}^{n} \sum_{k=0}^{n}\left(\begin{array}{l}
n \\
k
\end{array}\right) \sum_{i=0}^{w_{1}-1}(-1)^{i} E_{k}\left(y_{1}+\frac{i}{w_{1}}\right) T_{n-k}\left(w_{2}-1\right) w_{2}^{k} \\
=w_{2}^{n} \sum_{k=0}^{n}\left(\begin{array}{l}
n \\
k
\end{array}\right) \sum_{i=0}^{w_{2}-1}(-1)^{i} E_{k}\left(y_{1}+\frac{i}{w_{2}}\right) T_{n-k}\left(w_{1}-1\right) w_{1}^{k} \\
=\left(w_{1} w_{2}\right)^{n} \sum_{i=0}^{w_{1}-1} \sum_{j=0}^{w_{2}-1}(-1)^{i+j} E_{n}\left(y_{1}+\frac{i}{w_{1}}+\frac{j}{w_{2}}\right) .
\end{aligned}
$$

The derivations of identities will be based on the $p$-adic integral expression of the generating function for the Euler polynomials in (1.5) and the quotient of integrals in (1.8) that can be expressed as the exponential generating function for the alternating power sums. We indebted this idea to the paper in [3].

\section{Several Types of Quotients of Fermionic Integrals}

Here we will introduce several types of quotients of $p$-adic fermionic integrals on $\mathbb{Z}_{p}$ or $\mathbb{Z}_{p}^{3}$ from which some interesting identities follow owing to the built-in symmetries in $w_{1}, w_{2}$, $w_{3}$. In the following, $w_{1}, w_{2}, w_{3}$ are all positive integers and all of the explicit expressions of integrals in (2.2), (2.4), (2.6), and (2.8) are obtained from the identity in (1.4). 
(a) Type $\Lambda_{23}^{i}$ (for $\left.i=0,1,2,3\right)$. One has

$$
\begin{aligned}
I\left(\Lambda_{23}^{i}\right) & =\frac{\int_{\mathbb{Z}_{p}^{3}} e^{\left(w_{2} w_{3} x_{1}+w_{1} w_{3} x_{2}+w_{1} w_{2} x_{3}+w_{1} w_{2} w_{3}\left(\sum_{j=1}^{3-i} y_{j}\right)\right) t} d \mu_{-1}\left(x_{1}\right) d \mu_{-1}\left(x_{2}\right) d \mu_{-1}\left(x_{3}\right)}{\left(\int_{\mathbb{Z}_{p}} e^{w_{1} w_{2} w_{3} x_{4} t} d \mu_{-1}\left(x_{4}\right)\right)^{i}} \\
& =\frac{2^{3-i} e^{w_{1} w_{2} w_{3}\left(\sum_{j=1}^{3-i} y_{j}\right) t}\left(e^{w_{1} w_{2} w_{3} t}+1\right)^{i}}{\left(e^{w_{2} w_{3} t}+1\right)\left(e^{w_{1} w_{3} t}+1\right)\left(e^{w_{1} w_{2} t}+1\right)}
\end{aligned}
$$

(b) Type $\Lambda_{13}^{i}($ for $i=0,1,2,3)$. One has

$$
\begin{aligned}
I\left(\Lambda_{13}^{i}\right) & =\frac{\int_{\mathbb{Z}_{p}^{3}} e^{\left(w_{1} x_{1}+w_{2} x_{2}+w_{3} x_{3}+w_{1} w_{2} w_{3}\left(\sum_{j=1}^{3-i} y_{j}\right)\right) t} d \mu_{-1}\left(x_{1}\right) d \mu_{-1}\left(x_{2}\right) d \mu_{-1}\left(x_{3}\right)}{\left(\int_{\mathbb{Z}_{p}} e^{w_{1} w_{2} w_{3} x_{4} t} d \mu_{-1}\left(x_{4}\right)\right)^{i}} \\
& =\frac{2^{3-i} e^{w_{1} w_{2} w_{3}\left(\sum_{j=1}^{3-i} y_{j}\right) t}\left(e^{w_{1} w_{2} w_{3} t}+1\right)^{i}}{\left(e^{w_{1} t}+1\right)\left(e^{w_{2} t}+1\right)\left(e^{w_{3} t}+1\right)}
\end{aligned}
$$

(c-0) Type $\Lambda_{12}^{0}$. One has

$$
\begin{aligned}
I\left(\Lambda_{12}^{0}\right) & =\int_{\mathbb{Z}_{p}^{3}} e^{\left(w_{1} x_{1}+w_{2} x_{2}+w_{3} x_{3}+w_{2} w_{3} y+w_{1} w_{3} y+w_{1} w_{2} y\right) t} d \mu_{-1}\left(x_{1}\right) d \mu_{-1}\left(x_{2}\right) d \mu_{-1}\left(x_{3}\right) \\
& =\frac{8 e^{\left(w_{2} w_{3}+w_{1} w_{3}+w_{1} w_{2}\right) y t}}{\left(e^{w_{1} t}+1\right)\left(e^{w_{2} t}+1\right)\left(e^{w_{3} t}+1\right)}
\end{aligned}
$$

(c-1) Type $\Lambda_{12}^{1}$. One has

$$
\begin{aligned}
I\left(\Lambda_{12}^{1}\right) & =\frac{\int_{\mathbb{Z}_{p}^{3}} e^{\left(w_{1} x_{1}+w_{2} x_{2}+w_{3} x_{3}\right) t} d \mu_{-1}\left(x_{1}\right) d \mu_{-1}\left(x_{2}\right) d \mu_{-1}\left(x_{3}\right)}{\int_{\mathbb{Z}_{p}^{3}} e^{\left(w_{2} w_{3} z_{1}+w_{1} w_{3} z_{2}+w_{1} w_{2} z_{3}\right) t} d \mu_{-1}\left(z_{1}\right) d \mu_{-1}\left(z_{2}\right) d \mu_{-1}\left(z_{3}\right)} \\
& =\frac{\left(e^{w_{2} w_{3} t}+1\right)\left(e^{w_{1} w_{3} t}+1\right)\left(e^{w_{1} w_{2} t}+1\right)}{\left(e^{w_{1} t}+1\right)\left(e^{w_{2} t}+1\right)\left(e^{w_{3} t}+1\right)} .
\end{aligned}
$$

All of the above $p$-adic integrals of various types are invariant under all permutations of $w_{1}, w_{2}, w_{3}$ as one can see either from $p$-adic integral representations in $(2.1),(2.3),(2.5)$, and (2.7) or from their explicit evaluations in (2.2), (2.4), (2.6), and (2.8).

\section{Identities for Euler Polynomials}

In the following $w_{1}, w_{2}, w_{3}$ are all odd positive integers except for $(\mathrm{a}-0)$ and $(\mathrm{c}-0)$, where they are any positive integers. 
(a-0) First, let us consider Type $\Lambda_{23}^{i}$, for each $i=0,1,2,3$. The following results can be easily obtained from (1.5) and (1.8):

$$
\begin{aligned}
I\left(\Lambda_{23}^{0}\right) & =\int_{\mathbb{Z}_{p}} e^{w_{2} w_{3}\left(x_{1}+w_{1} y_{1}\right) t} d \mu_{-1}\left(x_{1}\right) \int_{\mathbb{Z}_{p}} e^{w_{1} w_{3}\left(x_{2}+w_{2} y_{2}\right) t} d \mu_{-1}\left(x_{2}\right) \int_{\mathbb{Z}_{p}} e^{w_{1} w_{2}\left(x_{3}+w_{3} y_{3}\right) t} d \mu_{-1}\left(x_{3}\right) \\
& =\left(\sum_{k=0}^{\infty} \frac{E_{k}\left(w_{1} y_{1}\right)}{k !}\left(w_{2} w_{3} t\right)^{k}\right)\left(\sum_{l=0}^{\infty} \frac{E_{l}\left(w_{2} y_{2}\right)}{l !}\left(w_{1} w_{3} t\right)^{l}\right)\left(\sum_{m=0}^{\infty} \frac{E_{m}\left(w_{3} y_{3}\right)}{m !}\left(w_{1} w_{2} t\right)^{m}\right) \\
& \left.=\sum_{n=0}^{\infty}\left(\begin{array}{c}
n \\
k+l+m=n \\
k, l, m
\end{array}\right) E_{k}\left(\begin{array}{c}
n \\
k \\
k
\end{array} y_{1}\right) E_{l}\left(w_{2} y_{2}\right) E_{m}\left(w_{3} y_{3}\right) w_{1}^{l+m} w_{2}^{k+m} w_{3}^{k+l}\right) \frac{t^{n}}{n !}
\end{aligned}
$$

where the inner sum is over all nonnegative integers $k, l, m$, with $k+l+m=n$, and

$$
\left(\begin{array}{c}
n \\
k, l, m
\end{array}\right)=\frac{n !}{k ! l ! m !} .
$$

(a-1) Here we write $I\left(\Lambda_{23}^{1}\right)$ in two different ways:

(1) One has

$$
\begin{aligned}
I\left(\Lambda_{23}^{1}\right)= & \int_{\mathbb{Z}_{p}} e^{w_{2} w_{3}\left(x_{1}+w_{1} y_{1}\right) t} d \mu_{-1}\left(x_{1}\right) \\
& \times \int_{\mathbb{Z}_{p}} e^{w_{1} w_{3}\left(x_{2}+w_{2} y_{2}\right) t} d \mu_{-1}\left(x_{2}\right) \times \frac{\int_{\mathbb{Z}_{p}} e^{w_{1} w_{2} x_{3} t} d \mu_{-1}\left(x_{3}\right)}{\int_{Z_{p}} e^{w_{1} w_{2} w_{3} x_{4} t} d \mu_{-1}\left(x_{4}\right)} \\
= & \left(\sum_{k=0}^{\infty} E_{k}\left(w_{1} y_{1}\right) \frac{\left(w_{2} w_{3} t\right)^{k}}{k !}\right)\left(\sum_{l=0}^{\infty} E_{l}\left(w_{2} y_{2}\right) \frac{\left(w_{1} w_{3} t\right)^{l}}{l !}\right)\left(\sum_{m=0}^{\infty} T_{m}\left(w_{3}-1\right) \frac{\left(w_{1} w_{2} t\right)^{m}}{m !}\right) \\
= & \left.\sum_{n=0}^{\infty}\left(\begin{array}{c}
n \\
k+l+m=n \\
k, l, m
\end{array}\right) E_{k}\left(w_{1} y_{1}\right) E_{l}\left(w_{2} y_{2}\right) T_{m}\left(w_{3}-1\right) w_{1}^{l+m} w_{2}^{k+m} w_{3}^{k+l}\right) \frac{t^{n}}{n !} .
\end{aligned}
$$

(2) Invoking (1.8), (3.3) can also be written as

$$
\begin{aligned}
I\left(\Lambda_{23}^{1}\right) & =\sum_{i=0}^{w_{3}-1}(-1)^{i} \int_{\mathbb{Z}_{p}} e^{w_{2} w_{3}\left(x_{1}+w_{1} y_{1}\right) t} d \mu_{-1}\left(x_{1}\right) \int_{\mathbb{Z}_{p}} e^{w_{1} w_{3}\left(x_{2}+w_{2} y_{2}+\left(w_{2} / w_{3}\right) i\right) t} d \mu_{-1}\left(x_{2}\right) \\
& =\sum_{i=0}^{w_{3}-1}(-1)^{i}\left(\sum_{k=0}^{\infty} E_{k}\left(w_{1} y_{1}\right) \frac{\left(w_{2} w_{3} t\right)^{k}}{k !}\right)\left(\sum_{l=0}^{\infty} E_{l}\left(w_{2} y_{2}+\frac{w_{2}}{w_{3}} i\right) \frac{\left(w_{1} w_{3} t\right)^{l}}{l !}\right) \\
& =\sum_{n=0}^{\infty}\left(w_{3}^{n} \sum_{k=0}^{n}\left(\begin{array}{l}
n \\
k
\end{array}\right) E_{k}\left(w_{1} y_{1}\right) \sum_{i=0}^{w_{3}-1}(-1)^{i} E_{n-k}\left(w_{2} y_{2}+\frac{w_{2}}{w_{3}} i\right) w_{1}^{n-k} w_{2}^{k}\right) \frac{t^{n}}{n !}
\end{aligned}
$$


(a-2) Here we write $I\left(\Lambda_{23}^{2}\right)$ in three different ways:

(1) One has

$$
\begin{aligned}
I\left(\Lambda_{23}^{2}\right)= & \int_{\mathbb{Z}_{p}} e^{w_{2} w_{3}\left(x_{1}+w_{1} y_{1}\right) t} d \mu_{-1}\left(x_{1}\right) \\
& \times \frac{\int_{\mathbb{Z}_{p}} e^{w_{1} w_{3} x_{2} t} d \mu_{-1}\left(x_{2}\right)}{\int_{\mathbb{Z}_{p}} e^{w_{1} w_{2} w_{3} x_{4} t} d \mu_{-1}\left(x_{4}\right)} \times \frac{\int_{\mathbb{Z}_{p}} e^{w_{1} w_{2} x_{3} t} d \mu_{-1}\left(x_{3}\right)}{\int_{\mathbb{Z}_{p}} e^{w_{1} w_{2} w_{3} x_{4} t} d \mu_{-1}\left(x_{4}\right)} \\
= & \left(\sum_{k=0}^{\infty} E_{k}\left(w_{1} y_{1}\right) \frac{\left(w_{2} w_{3} t\right)^{k}}{k !}\right)\left(\sum_{l=0}^{\infty} T_{l}\left(w_{2}-1\right) \frac{\left(w_{1} w_{3} t\right)^{l}}{l !}\right)\left(\sum_{m=0}^{\infty} T_{m}\left(w_{3}-1\right) \frac{\left(w_{1} w_{2} t\right)^{m}}{m !}\right) \\
= & \sum_{n=0}^{\infty}\left(\sum_{k+l+m=n}\left(\begin{array}{c}
n \\
k, l, m
\end{array}\right) E_{k}\left(w_{1} y_{1}\right) T_{l}\left(w_{2}-1\right) T_{m}\left(w_{3}-1\right) w_{1}^{l+m} w_{2}^{k+m} w_{3}^{k+l}\right) \frac{t^{n}}{n !} .
\end{aligned}
$$

(2) Invoking (1.8), (3.6) can also be written as

$$
\begin{aligned}
I\left(\Lambda_{23}^{2}\right) & =\sum_{i=0}^{w_{2}-1}(-1)^{i} \int_{\mathbb{Z}_{p}} e^{w_{2} w_{3}\left(x_{1}+w_{1} y_{1}+\left(w_{1} / w_{2}\right) i\right) t} d \mu_{-1}\left(x_{1}\right) \times \frac{\int_{\mathbb{Z}_{p}} e^{w_{1} w_{2} x_{3} t} d \mu_{-1}\left(x_{3}\right)}{\int_{\mathbb{Z}_{p}} e^{w_{1} w_{2} w_{3} x_{4} t} d \mu_{-1}\left(x_{4}\right)} \\
& =\sum_{i=0}^{w_{2}-1}(-1)^{i}\left(\sum_{k=0}^{\infty} E_{k}\left(w_{1} y_{1}+\frac{w_{1}}{w_{2}} i\right) \frac{\left(w_{2} w_{3} t\right)^{k}}{k !}\right)\left(\sum_{l=0}^{\infty} T_{l}\left(w_{3}-1\right) \frac{\left(w_{1} w_{2} t\right)^{l}}{l !}\right) \\
& =\sum_{n=0}^{\infty}\left(w_{2}^{n} \sum_{k=0}^{n}\left(\begin{array}{l}
n \\
k
\end{array}\right) \sum_{i=0}^{w_{2}-1}(-1)^{i} E_{k}\left(w_{1} y_{1}+\frac{w_{1}}{w_{2}} i\right) T_{n-k}\left(w_{3}-1\right) w_{1}^{n-k} w_{3}^{k}\right) \frac{t^{n}}{n !}
\end{aligned}
$$

(3) Invoking (1.8) once again, (3.8) can be written as

$$
\begin{aligned}
I\left(\Lambda_{23}^{2}\right) & =\sum_{i=0}^{w_{2}-1} \sum_{j=0}^{w_{3}-1}(-1)^{i+j} \int_{\mathbb{Z}_{p}} e^{w_{2} w_{3}\left(x_{1}+w_{1} y_{1}+\left(w_{1} / w_{2}\right) i+\left(w_{1} / w_{3}\right) j\right) t} d \mu_{-1}\left(x_{1}\right) \\
& =\sum_{i=0}^{w_{2}-1} \sum_{j=0}^{w_{3}-1}(-1)^{i+j} \sum_{n=0}^{\infty} E_{n}\left(w_{1} y_{1}+\frac{w_{1}}{w_{2}} i+\frac{w_{1}}{w_{3}} j\right) \frac{\left(w_{2} w_{3} t\right)^{n}}{n !} \\
& =\sum_{n=0}^{\infty}\left(\left(w_{2} w_{3}\right)^{n} \sum_{i=0}^{w_{2}-1} \sum_{j=0}^{w_{3}-1}(-1)^{i+j} E_{n}\left(w_{1} y_{1}+\frac{w_{1}}{w_{2}} i+\frac{w_{1}}{w_{3}} j\right)\right) \frac{t^{n}}{n !}
\end{aligned}
$$


(a-3) One has

$$
\begin{aligned}
I\left(\Lambda_{23}^{3}\right) & =\frac{\int_{\mathbb{Z}_{p}} e^{w_{2} w_{3} x_{1} t} d \mu_{-1}\left(x_{1}\right)}{\int_{\mathbb{Z}_{p}} e^{w_{1} w_{2} w_{3} x_{4} t} d \mu_{-1}\left(x_{4}\right)} \times \frac{\int_{\mathbb{Z}_{p}} e^{w_{1} w_{3} x_{2} t} d \mu_{-1}\left(x_{2}\right)}{\int_{\mathbb{Z}_{p}} e^{w_{1} w_{2} w_{3} x_{4} t} d \mu_{-1}\left(x_{4}\right)} \times \frac{\int_{\mathbb{Z}_{p}} e^{w_{1} w_{2} x_{3} t} d \mu_{-1}\left(x_{3}\right)}{\int_{\mathbb{Z}_{p}} e^{w_{1} w_{2} w_{3} x_{4} t} d \mu_{-1}\left(x_{4}\right)} \\
& =\left(\sum_{k=0}^{\infty} T_{k}\left(w_{1}-1\right) \frac{\left(w_{2} w_{3} t\right)^{k}}{k !}\right)\left(\sum_{l=0}^{\infty} T_{l}\left(w_{2}-1\right) \frac{\left(w_{1} w_{3} t\right)^{l}}{l !}\right)\left(\sum_{m=0}^{\infty} T_{m}\left(w_{3}-1\right) \frac{\left(w_{1} w_{2} t\right)^{m}}{m !}\right) \\
& =\sum_{n=0}^{\infty} \sum_{k+l+m=n}\left(\left(\begin{array}{c}
n \\
k, l, m
\end{array}\right) T_{k}\left(w_{1}-1\right) T_{l}\left(w_{2}-1\right) T_{m}\left(w_{3}-1\right) w_{1}^{l+m} w_{2}^{k+m} w_{3}^{k+l}\right) \frac{t^{n}}{n !} .
\end{aligned}
$$

(b) For Type $\Lambda_{13}^{i}(i=0,1,2,3)$, we may consider the analogous things to the ones in (a$0),(a-1),(a-2)$, and (a-3). However, these do not lead us to new identities. Indeed, if we substitute $w_{2} w_{3}, w_{1} w_{3}, w_{1} w_{2}$, respectively, for $w_{1}, w_{2}, w_{3}$ in (2.1), this amounts to replacing $t$ by $w_{1} w_{2} w_{3} t$ in (2.3). So, upon replacing $w_{1}, w_{2}, w_{3}$, respectively, by $w_{2} w_{3}, w_{1} w_{3}, w_{1} w_{2}$, and then dividing by $\left(w_{1} w_{2} w_{3}\right)^{n}$, in each of the expressions of Theorem 4.1 through Corollary 4.15 , we will get the corresponding symmetric identities for Type $\Lambda_{13}^{i}(i=0,1,2,3)$.

(c-0) One has

$$
\begin{aligned}
I\left(\Lambda_{12}^{0}\right) & =\int_{\mathbb{Z}_{p}} e^{w_{1}\left(x_{1}+w_{2} y\right) t} d \mu_{-1}\left(x_{1}\right) \int_{\mathbb{Z}_{p}} e^{w_{2}\left(x_{2}+w_{3} y\right) t} d \mu_{-1}\left(x_{2}\right) \int_{\mathbb{Z}_{p}} e^{w_{3}\left(x_{3}+w_{1} y\right) t} d \mu_{-1}\left(x_{3}\right) \\
& =\left(\sum_{n=0}^{\infty} \frac{E_{k}\left(w_{2} y\right)}{k !}\left(w_{1} t\right)^{k}\right)\left(\sum_{l=0}^{\infty} \frac{E_{l}\left(w_{3} y\right)}{l !}\left(w_{2} t\right)^{l}\right)\left(\sum_{m=0}^{\infty} \frac{E_{m}\left(w_{1} y\right)}{m !}\left(w_{3} t\right)^{m}\right) \\
& =\sum_{n=0}^{\infty}\left(\sum_{k+l+m=n}\left(\begin{array}{c}
n \\
k, l, m
\end{array}\right) E_{k}\left(w_{2} y\right) E_{l}\left(w_{3} y\right) E_{m}\left(w_{1} y\right) w_{1}^{k} w_{2}^{l} w_{3}^{m}\right) \frac{t^{n}}{n !}
\end{aligned}
$$

(c-1) One has

$$
\begin{aligned}
& \frac{\int_{\mathbb{Z}_{p}} e^{w_{1} x_{1} t} d \mu_{-1}\left(x_{1}\right)}{\int_{\mathbb{Z}_{p}} e^{w_{1} w_{2} z_{3} t} d \mu_{-1}\left(z_{3}\right)} \times \frac{\int_{\mathbb{Z}_{p}} e^{w_{2} x_{2} t} d \mu_{-1}\left(x_{2}\right)}{\int_{\mathbb{Z}_{p}} e^{w_{2} w_{3} z_{1} t} d \mu_{-1}\left(z_{1}\right)} \times \frac{\int_{\mathbb{Z}_{p}} e^{w_{3} x_{3} t} d \mu_{-1}\left(x_{3}\right)}{\int_{\mathbb{Z}_{p}} e^{w_{3} w_{1} z_{2} t} d \mu_{-1}\left(z_{2}\right)} \\
& =\left(\sum_{k=0}^{\infty} T_{k}\left(w_{2}-1\right) \frac{\left(w_{1} t\right)^{k}}{k !}\right)\left(\sum_{l=0}^{\infty} T_{l}\left(w_{3}-1\right) \frac{\left(w_{2} t\right)^{l}}{l !}\right)\left(\sum_{m=0}^{\infty} T_{m}\left(w_{1}-1\right) \frac{\left(w_{3} t\right)^{m}}{m !}\right) \\
& =\sum_{n=0}^{\infty}\left(\sum_{k+l+m=n}\left(\begin{array}{c}
n \\
k, l, m
\end{array}\right) T_{k}\left(w_{2}-1\right) T_{l}\left(w_{3}-1\right) T_{m}\left(w_{1}-1\right) w_{1}^{k} w_{2}^{l} w_{3}^{m}\right) \frac{t^{n}}{n !} .
\end{aligned}
$$




\section{Main Theorems}

As we noted earlier in the last paragraph of Section 2, the various types of quotients of $p$-adic fermionic integrals are invariant under any permutation of $w_{1}, w_{2}, w_{3}$. So the corresponding expressions in Section 3 are also invariant under any permutation of $w_{1}, w_{2}, w_{3}$. Thus our results about identities of symmetry will be immediate consequences of this observation.

However, not all permutations of an expression in Section 3 yield distinct ones. In fact, as these expressions are obtained by permuting $w_{1}, w_{2}, w_{3}$ in a single one labelled by them, they can be viewed as a group in a natural manner and hence it is isomorphic to a quotient of $S_{3}$. In particular, the numbers of possible distinct expressions are 1, 2, 3, or 6. (a-0), (a$1(1)),(a-1(2))$, and (a-2(2)) give the full six identities of symmetry, (a-2(1)) and (a-2(3)) yield three identities of symmetry, and (c-0) and (c-1) give two identities of symmetry, while the expression in (a-3) yields no identities of symmetry.

Here we will just consider the cases of Theorems 4.8 and 4.17 leaving the others as easy exercises for the reader. As for the case of Theorem 4.8, in addition to (4.15)-(4.17), we get the following three ones:

$$
\begin{gathered}
\sum_{k+l+m=n}\left(\begin{array}{c}
n \\
k, l, m
\end{array}\right) E_{k}\left(w_{1} y_{1}\right) T_{l}\left(w_{3}-1\right) T_{m}\left(w_{2}-1\right) w_{1}^{l+m} w_{3}^{k+m} w_{2}^{k+l}, \\
\sum_{k+l+m=n}\left(\begin{array}{c}
n \\
k, l, m
\end{array}\right) E_{k}\left(w_{2} y_{1}\right) T_{l}\left(w_{1}-1\right) T_{m}\left(w_{3}-1\right) w_{2}^{l+m} w_{1}^{k+m} w_{3}^{k+l}, \\
\sum_{k+l+m=n}\left(\begin{array}{c}
n \\
k, l, m
\end{array}\right) E_{k}\left(w_{3} y_{1}\right) T_{l}\left(w_{2}-1\right) T_{m}\left(w_{1}-1\right) w_{3}^{l+m} w_{2}^{k+m} w_{1}^{k+l} .
\end{gathered}
$$

But, by interchanging $l$ and $m$, we see that (4.1), (4.2), and (4.3) are, respectively, equal to (4.15), (4.16), and (4.17).

As to Theorem 17, in addition to (4.26) and (4.27), we have

$$
\begin{gathered}
\sum_{k+l+m=n}\left(\begin{array}{c}
n \\
k, l, m
\end{array}\right) T_{k}\left(w_{2}-1\right) T_{l}\left(w_{3}-1\right) T_{m}\left(w_{1}-1\right) w_{1}^{k} w_{2}^{l} w_{3}^{m}, \\
\sum_{k+l+m=n}\left(\begin{array}{c}
n \\
k, l, m
\end{array}\right) T_{k}\left(w_{3}-1\right) T_{l}\left(w_{1}-1\right) T_{m}\left(w_{2}-1\right) w_{2}^{k} w_{3}^{l} w_{1}^{m}, \\
\sum_{k+l+m=n}\left(\begin{array}{c}
n \\
k, l, m
\end{array}\right) T_{k}\left(w_{3}-1\right) T_{l}\left(w_{2}-1\right) T_{m}\left(w_{1}-1\right) w_{1}^{k} w_{3}^{l} w_{2}^{m}, \\
\sum_{k+l+m=n}\left(\begin{array}{c}
n \\
k, l, m
\end{array}\right) T_{k}\left(w_{2}-1\right) T_{l}\left(w_{1}-1\right) T_{m}\left(w_{3}-1\right) w_{3}^{k} w_{2}^{l} w_{1}^{m} .
\end{gathered}
$$

However, (4.4) and (4.5) are equal to (4.26), as we can see by applying the permutations $k \rightarrow l, l \rightarrow m$, and $m \rightarrow k$ for (4.4) and $k \rightarrow m, l \rightarrow k$, and $m \rightarrow l$ for (4.5). Similarly, 
Journal of Inequalities and Applications

we see that (4.6) and (4.7) are equal to (4.27), by applying permutations $k \rightarrow l, l \rightarrow m$, and $m \rightarrow k$ for (4.6) and $k \rightarrow m, l \rightarrow k$, and $m \rightarrow l$ for (4.7).

Theorem 4.1. Let $w_{1}, w_{2}, w_{3}$ be any positive integers. Then the following expression is invariant under any permutation of $w_{1}, w_{2}, w_{3}$, so that it gives us six symmetries:

$$
\begin{aligned}
& \sum_{k+l+m=n}\left(\begin{array}{c}
n \\
k, l, m
\end{array}\right) E_{k}\left(w_{1} y_{1}\right) E_{l}\left(w_{2} y_{2}\right) E_{m}\left(w_{3} y_{3}\right) w_{1}^{l+m} w_{2}^{k+m} w_{3}^{k+l} \\
& =\sum_{k+l+m=n}\left(\begin{array}{c}
n \\
k, l, m
\end{array}\right) E_{k}\left(w_{1} y_{1}\right) E_{l}\left(w_{3} y_{2}\right) E_{m}\left(w_{2} y_{3}\right) w_{1}^{l+m} w_{3}^{k+m} w_{2}^{k+l} \\
& =\sum_{k+l+m=n}\left(\begin{array}{c}
n \\
k, l, m
\end{array}\right) E_{k}\left(w_{2} y_{1}\right) E_{l}\left(w_{1} y_{2}\right) E_{m}\left(w_{3} y_{3}\right) w_{2}^{l+m} w_{1}^{k+m} w_{3}^{k+l} \\
& =\sum_{k+l+m=n}\left(\begin{array}{c}
n \\
k, l, m
\end{array}\right) E_{k}\left(w_{2} y_{1}\right) E_{l}\left(w_{3} y_{2}\right) E_{m}\left(w_{1} y_{3}\right) w_{2}^{l+m} w_{3}^{k+m} w_{1}^{k+l} \\
& =\sum_{k+l+m=n}\left(\begin{array}{c}
n \\
k, l, m
\end{array}\right) E_{k}\left(w_{3} y_{1}\right) E_{l}\left(w_{1} y_{2}\right) E_{m}\left(w_{2} y_{3}\right) w_{3}^{l+m} w_{1}^{k+m} w_{2}^{k+l} \\
& =\sum_{k+l+m=n}\left(\begin{array}{c}
n \\
k, l, m
\end{array}\right) E_{k}\left(w_{3} y_{1}\right) E_{l}\left(w_{2} y_{2}\right) E_{m}\left(w_{1} y_{3}\right) w_{3}^{l+m} w_{2}^{k+m} w_{1}^{k+l} .
\end{aligned}
$$

Theorem 4.2. Let $w_{1}, w_{2}, w_{3}$ be any odd positive integers. Then the following expression is invariant under any permutation of $w_{1}, w_{2}, w_{3}$, so that it gives us six symmetries:

$$
\begin{aligned}
& \sum_{k+l+m=n}\left(\begin{array}{c}
n \\
k, l, m
\end{array}\right) E_{k}\left(w_{1} y_{1}\right) E_{l}\left(w_{2} y_{2}\right) T_{m}\left(w_{3}-1\right) w_{1}^{l+m} w_{2}^{k+m} w_{3}^{k+l} \\
& =\sum_{k+l+m=n}\left(\begin{array}{c}
n \\
k, l, m
\end{array}\right) E_{k}\left(w_{1} y_{1}\right) E_{l}\left(w_{3} y_{2}\right) T_{m}\left(w_{2}-1\right) w_{1}^{l+m} w_{3}^{k+m} w_{2}^{k+l} \\
& =\sum_{k+l+m=n}\left(\begin{array}{c}
n \\
k, l, m
\end{array}\right) E_{k}\left(w_{2} y_{1}\right) E_{l}\left(w_{1} y_{2}\right) T_{m}\left(w_{3}-1\right) w_{2}^{l+m} w_{1}^{k+m} w_{3}^{k+l} \\
& =\sum_{k+l+m=n}\left(\begin{array}{c}
n \\
k, l, m
\end{array}\right) E_{k}\left(w_{2} y_{1}\right) E_{l}\left(w_{3} y_{2}\right) T_{m}\left(w_{1}-1\right) w_{2}^{l+m} w_{3}^{k+m} w_{1}^{k+l} \\
& =\sum_{k+l+m=n}\left(\begin{array}{c}
n \\
k, l, m
\end{array}\right) E_{k}\left(w_{3} y_{1}\right) E_{l}\left(w_{2} y_{2}\right) T_{m}\left(w_{1}-1\right) w_{3}^{l+m} w_{2}^{k+m} w_{1}^{k+l} \\
& =\sum_{k+l+m=n}\left(\begin{array}{c}
n \\
k, l, m
\end{array}\right) E_{k}\left(w_{3} y_{1}\right) E_{l}\left(w_{1} y_{2}\right) T_{m}\left(w_{2}-1\right) w_{3}^{l+m} w_{1}^{k+m} w_{2}^{k+l} .
\end{aligned}
$$


Putting $w_{3}=1$ in (4.9), we get the following corollary.

Corollary 4.3. Let $w_{1}, w_{2}$ be any odd positive integers. Then one has

$$
\begin{aligned}
\sum_{k=0}^{n}\left(\begin{array}{l}
n \\
k
\end{array}\right) E_{k}\left(w_{1} y_{1}\right) E_{n-k}\left(w_{2} y_{2}\right) w_{1}^{n-k} w_{2}^{k} \\
=\sum_{k=0}^{n}\left(\begin{array}{l}
n \\
k
\end{array}\right) E_{k}\left(w_{2} y_{1}\right) E_{n-k}\left(w_{1} y_{2}\right) w_{2}^{n-k} w_{1}^{k} \\
=\sum_{k+l+m=n}\left(\begin{array}{c}
n \\
k, l, m
\end{array}\right) E_{k}\left(y_{1}\right) E_{l}\left(w_{2} y_{2}\right) T_{m}\left(w_{1}-1\right) w_{2}^{k+m} w_{1}^{k+l} \\
=\sum_{k+l+m=n}\left(\begin{array}{c}
n \\
k, l, m
\end{array}\right) E_{k}\left(w_{2} y_{1}\right) E_{l}\left(y_{2}\right) T_{m}\left(w_{1}-1\right) w_{2}^{l+m} w_{1}^{k+l} \\
=\sum_{k+l+m=n}\left(\begin{array}{c}
n \\
k, l, m
\end{array}\right) E_{k}\left(y_{1}\right) E_{l}\left(w_{1} y_{2}\right) T_{m}\left(w_{2}-1\right) w_{1}^{k+m} w_{2}^{k+l} \\
=\sum_{k+l+m=n}\left(\begin{array}{c}
n \\
k, l, m
\end{array}\right) E_{k}\left(w_{1} y_{1}\right) E_{l}\left(y_{2}\right) T_{m}\left(w_{2}-1\right) w_{1}^{l+m} w_{2}^{k+l}
\end{aligned}
$$

Letting further $w_{2}=1$ in (4.10), we have the following corollary.

Corollary 4.4. Let $w_{1}$ be any odd positive integer. Then one has

$$
\begin{aligned}
\sum_{k=0}^{n}\left(\begin{array}{l}
n \\
k
\end{array}\right) E_{k}\left(w_{1} y_{1}\right) E_{n-k}\left(y_{2}\right) w_{1}^{n-k} \\
\quad=\sum_{k=0}^{n}\left(\begin{array}{l}
n \\
k
\end{array}\right) E_{k}\left(y_{1}\right) E_{n-k}\left(w_{1} y_{2}\right) w_{1}^{k} \\
=\sum_{k+l+m=n}\left(\begin{array}{c}
n \\
k, l, m
\end{array}\right) E_{k}\left(y_{1}\right) E_{l}\left(y_{2}\right) T_{m}\left(w_{1}-1\right) w_{1}^{k+l} .
\end{aligned}
$$


Theorem 4.5. Let $w_{1}, w_{2}, w_{3}$ be any odd positive integers. Then the following expression is invariant under any permutation of $w_{1}, w_{2}, w_{3}$, so that it gives us six symmetries:

$$
\begin{aligned}
w_{1}^{n} \sum_{k=0}^{n}\left(\begin{array}{l}
n \\
k
\end{array}\right) E_{k}\left(w_{3} y_{1}\right) \sum_{i=0}^{w_{1}-1}(-1)^{i} E_{n-k}\left(w_{2} y_{2}+\frac{w_{2}}{w_{1}} i\right) w_{3}^{n-k} w_{2}^{k} \\
=w_{1}^{n} \sum_{k=0}^{n}\left(\begin{array}{l}
n \\
k
\end{array}\right) E_{k}\left(w_{2} y_{1}\right) \sum_{i=0}^{w_{1}-1}(-1)^{i} E_{n-k}\left(w_{3} y_{2}+\frac{w_{3}}{w_{1}} i\right) w_{2}^{n-k} w_{3}^{k} \\
=w_{2}^{n} \sum_{k=0}^{n}\left(\begin{array}{l}
n \\
k
\end{array}\right) E_{k}\left(w_{3} y_{1}\right) \sum_{i=0}^{w_{2}-1}(-1)^{i} E_{n-k}\left(w_{1} y_{2}+\frac{w_{1}}{w_{2}} i\right) w_{3}^{n-k} w_{1}^{k} \\
=w_{2}^{n} \sum_{k=0}^{n}\left(\begin{array}{l}
n \\
k
\end{array}\right) E_{k}\left(w_{1} y_{1}\right) \sum_{i=0}^{w_{2}-1}(-1)^{i} E_{n-k}\left(w_{3} y_{2}+\frac{w_{3}}{w_{2}} i\right) w_{1}^{n-k} w_{3}^{k} \\
=w_{3}^{n} \sum_{k=0}^{n}\left(\begin{array}{l}
n \\
k
\end{array}\right) E_{k}\left(w_{2} y_{1}\right) \sum_{i=0}^{w_{3}-1}(-1)^{i} E_{n-k}\left(w_{1} y_{2}+\frac{w_{1}}{w_{3}} i\right) w_{2}^{n-k} w_{1}^{k} \\
=w_{3}^{n} \sum_{k=0}^{n}\left(\begin{array}{l}
n \\
k
\end{array}\right) E_{k}\left(w_{1} y_{1}\right) \sum_{i=0}^{w_{3}-1}(-1)^{i} E_{n-k}\left(w_{2} y_{2}+\frac{w_{2}}{w_{3}} i\right) w_{1}^{n-k} w_{2}^{k}
\end{aligned}
$$

Letting $w_{3}=1$ in (4.12), we obtain alternative expressions for the identities in (4.10).

Corollary 4.6. Let $w_{1}, w_{2}$ be any odd positive integers. Then one has

$$
\begin{aligned}
\sum_{k=0}^{n}\left(\begin{array}{l}
n \\
k
\end{array}\right) E_{k}\left(w_{1} y_{1}\right) E_{n-k}\left(w_{2} y_{2}\right) w_{1}^{n-k} w_{2}^{k} \\
=\sum_{k=0}^{n}\left(\begin{array}{l}
n \\
k
\end{array}\right) E_{k}\left(w_{2} y_{1}\right) E_{n-k}\left(w_{1} y_{2}\right) w_{2}^{n-k} w_{1}^{k} \\
=w_{1}^{n} \sum_{k=0}^{n}\left(\begin{array}{l}
n \\
k
\end{array}\right) E_{k}\left(y_{1}\right) \sum_{i=0}^{w_{1}-1}(-1)^{i} E_{n-k}\left(w_{2} y_{2}+\frac{w_{2}}{w_{1}} i\right) w_{2}^{k} \\
=w_{1}^{n} \sum_{k=0}^{n}\left(\begin{array}{l}
n \\
k
\end{array}\right) E_{k}\left(w_{2} y_{1}\right) \sum_{i=0}^{w_{1}-1}(-1)^{i} E_{n-k}\left(y_{2}+\frac{i}{w_{1}}\right) w_{2}^{n-k} \\
=w_{2}^{n} \sum_{k=0}^{n}\left(\begin{array}{l}
n \\
k
\end{array}\right) E_{k}\left(y_{1}\right) \sum_{i=0}^{w_{2}-1}(-1)^{i} E_{n-k}\left(w_{1} y_{2}+\frac{w_{1}}{w_{2}} i\right) w_{1}^{k} \\
=w_{2}^{n} \sum_{k=0}^{n}\left(\begin{array}{l}
n \\
k
\end{array}\right) E_{k}\left(w_{1} y_{1}\right) \sum_{i=0}^{w_{2}-1}(-1)^{i} E_{n-k}\left(y_{2}+\frac{i}{w_{2}}\right) w_{1}^{n-k}
\end{aligned}
$$
for (4.11).

Putting further $w_{2}=1$ in (4.13), we have the alternative expressions for the identities 
Corollary 4.7. Let $w_{1}$ be any odd positive integer. Then one has

$$
\begin{aligned}
\sum_{k=0}^{n}\left(\begin{array}{l}
n \\
k
\end{array}\right) E_{k}\left(y_{1}\right) E_{n-k}\left(w_{1} y_{2}\right) w_{1}^{k} \\
=\sum_{k=0}^{n}\left(\begin{array}{l}
n \\
k
\end{array}\right) E_{k}\left(y_{2}\right) E_{n-k}\left(w_{1} y_{1}\right) w_{1}^{k} \\
=w_{1}^{n} \sum_{k=0}^{n}\left(\begin{array}{l}
n \\
k
\end{array}\right) E_{k}\left(y_{1}\right) \sum_{i=0}^{w_{1}-1}(-1)^{i} E_{n-k}\left(y_{2}+\frac{i}{w_{1}}\right) .
\end{aligned}
$$

Theorem 4.8. Let $w_{1}, w_{2}, w_{3}$ be any odd positive integers. Then one has the following three symmetries in $w_{1}, w_{2}, w_{3}$ :

$$
\begin{aligned}
& \sum_{k+l+m=n}\left(\begin{array}{c}
n \\
k, l, m
\end{array}\right) E_{k}\left(w_{1} y_{1}\right) T_{l}\left(w_{2}-1\right) T_{m}\left(w_{3}-1\right) w_{1}^{l+m} w_{2}^{k+m} w_{3}^{k+l} \\
& =\sum_{k+l+m=n}\left(\begin{array}{c}
n \\
k, l, m
\end{array}\right) E_{k}\left(w_{2} y_{1}\right) T_{l}\left(w_{3}-1\right) T_{m}\left(w_{1}-1\right) w_{2}^{l+m} w_{3}^{k+m} w_{1}^{k+l} \\
& =\sum_{k+l+m=n}\left(\begin{array}{c}
n \\
k, l, m
\end{array}\right) E_{k}\left(w_{3} y_{1}\right) T_{l}\left(w_{1}-1\right) T_{m}\left(w_{2}-1\right) w_{3}^{l+m} w_{1}^{k+m} w_{2}^{k+l} .
\end{aligned}
$$

Putting $w_{3}=1$ in $(4.15)-(4.17)$, we get the following corollary.

Corollary 4.9. Let $w_{1}, w_{2}$ be any odd positive integers. Then one has

$$
\begin{aligned}
& \sum_{k=0}^{n}\left(\begin{array}{l}
n \\
k
\end{array}\right) E_{k}\left(w_{1} y_{1}\right) T_{n-k}\left(w_{2}-1\right) w_{1}^{n-k} w_{2}^{k} \\
& =\sum_{k=0}^{n}\left(\begin{array}{l}
n \\
k
\end{array}\right) E_{k}\left(w_{2} y_{1}\right) T_{n-k}\left(w_{1}-1\right) w_{2}^{n-k} w_{1}^{k} \\
& =\sum_{k+l+m=n}\left(\begin{array}{c}
n \\
k, l, m
\end{array}\right) E_{k}\left(y_{1}\right) T_{l}\left(w_{1}-1\right) T_{m}\left(w_{2}-1\right) w_{1}^{k+m} w_{2}^{k+l} .
\end{aligned}
$$

Letting further $w_{2}=1$ in (4.18), we get the following corollary. This is also obtained in [20, Corollary 2] and mentioned in [3].

Corollary 4.10. Let $w_{1}$ be any odd positive integer. Then one has

$$
E_{n}\left(w_{1} y_{1}\right)=\sum_{k=0}^{n}\left(\begin{array}{l}
n \\
k
\end{array}\right) E_{k}\left(y_{1}\right) T_{n-k}\left(w_{1}-1\right) w_{1}^{k}
$$


Theorem 4.11. Let $w_{1}, w_{2}, w_{3}$ be any odd positive integers. Then the following expression is invariant under any permutation of $w_{1}, w_{2}, w_{3}$, so that it gives us six symmetries:

$$
\begin{aligned}
w_{1}^{n} \sum_{k=0}^{n}\left(\begin{array}{l}
n \\
k
\end{array}\right) \sum_{i=0}^{w_{1}-1}(-1)^{i} E_{k}\left(w_{2} y_{1}+\frac{w_{2}}{w_{1}} i\right) T_{n-k}\left(w_{3}-1\right) w_{2}^{n-k} w_{3}^{k} \\
=w_{1}^{n} \sum_{k=0}^{n}\left(\begin{array}{l}
n \\
k
\end{array}\right) \sum_{i=0}^{w_{1}-1}(-1)^{i} E_{k}\left(w_{3} y_{1}+\frac{w_{3}}{w_{1}} i\right) T_{n-k}\left(w_{2}-1\right) w_{3}^{n-k} w_{2}^{k} \\
=w_{2}^{n} \sum_{k=0}^{n}\left(\begin{array}{l}
n \\
k
\end{array}\right) \sum_{i=0}^{w_{2}-1}(-1)^{i} E_{k}\left(w_{1} y_{1}+\frac{w_{1}}{w_{2}} i\right) T_{n-k}\left(w_{3}-1\right) w_{1}^{n-k} w_{3}^{k} \\
=w_{2}^{n} \sum_{k=0}^{n}\left(\begin{array}{l}
n \\
k
\end{array}\right) \sum_{i=0}^{w_{2}-1}(-1)^{i} E_{k}\left(w_{3} y_{1}+\frac{w_{3}}{w_{2}} i\right) T_{n-k}\left(w_{1}-1\right) w_{3}^{n-k} w_{1}^{k} \\
=w_{3}^{n} \sum_{k=0}^{n}\left(\begin{array}{l}
n \\
k
\end{array}\right) \sum_{i=0}^{w_{3}-1}(-1)^{i} E_{k}\left(w_{1} y_{1}+\frac{w_{1}}{w_{3}} i\right) T_{n-k}\left(w_{2}-1\right) w_{1}^{n-k} w_{2}^{k} \\
=w_{3}^{n} \sum_{k=0}^{n}\left(\begin{array}{l}
n \\
k
\end{array}\right) \sum_{i=0}^{w_{3}-1}(-1)^{i} E_{k}\left(w_{2} y_{1}+\frac{w_{2}}{w_{3}} i\right) T_{n-k}\left(w_{1}-1\right) w_{2}^{n-k} w_{1}^{k}
\end{aligned}
$$

Putting $w_{3}=1$ in (4.20), we obtain the following corollary. In Section 1, the identities in (4.18), (4.21), and (4.24) are combined to give those in (1.9)-(1.16).

Corollary 4.12. Let $w_{1}, w_{2}$ be any odd positive integers. Then one has

$$
\begin{aligned}
w_{1}^{n} \sum_{i=0}^{w_{1}-1}(-1)^{i} E_{n}\left(w_{2} y_{1}+\frac{w_{2}}{w_{1}} i\right) \\
=w_{2}^{n} \sum_{i=0}^{w_{2}-1}(-1)^{i} E_{n}\left(w_{1} y_{1}+\frac{w_{1}}{w_{2}} i\right) \\
=\sum_{k=0}^{n}\left(\begin{array}{l}
n \\
k
\end{array}\right) E_{k}\left(w_{2} y_{1}\right) T_{n-k}\left(w_{1}-1\right) w_{2}^{n-k} w_{1}^{k} \\
=\sum_{k=0}^{n}\left(\begin{array}{l}
n \\
k
\end{array}\right) E_{k}\left(w_{1} y_{1}\right) T_{n-k}\left(w_{2}-1\right) w_{1}^{n-k} w_{2}^{k} \\
=w_{1}^{n} \sum_{k=0}^{n}\left(\begin{array}{l}
n \\
k
\end{array}\right) \sum_{i=0}^{w_{1}-1}(-1)^{i} E_{k}\left(y_{1}+\frac{i}{w_{1}}\right) T_{n-k}\left(w_{2}-1\right) w_{2}^{k} \\
=w_{2}^{n} \sum_{k=0}^{n}\left(\begin{array}{l}
n \\
k
\end{array}\right) \sum_{i=0}^{w_{2}-1}(-1)^{i} E_{k}\left(y_{1}+\frac{i}{w_{2}}\right) T_{n-k}\left(w_{1}-1\right) w_{1}^{k} .
\end{aligned}
$$


Letting further $w_{2}=1$ in (4.21), we get the following corollary. This is the multiplication formula for Euler polynomials together with the relatively new identity mentioned in (4.19).

Corollary 4.13. Let $w_{1}$ be any odd positive integer. Then one has

$$
\begin{aligned}
E_{n}\left(w_{1} y_{1}\right) & =w_{1}^{n} \sum_{i=0}^{w_{1}-1}(-1)^{i} E_{n}\left(y_{1}+\frac{i}{w_{1}}\right) \\
& =\sum_{k=0}^{n}\left(\begin{array}{l}
n \\
k
\end{array}\right) E_{k}\left(y_{1}\right) T_{n-k}\left(w_{1}-1\right) w_{1}^{k} .
\end{aligned}
$$

Theorem 4.14. Let $w_{1}, w_{2}, w_{3}$ be any odd positive integers. Then one has the following three symmetries in $w_{1}, w_{2}, w_{3}$ :

$$
\begin{aligned}
& \left(w_{1} w_{2}\right)^{n} \sum_{i=0}^{w_{1}-1} \sum_{j=0}^{w_{2}-1}(-1)^{i+j} E_{n}\left(w_{3} y_{1}+\frac{w_{3}}{w_{1}} i+\frac{w_{3}}{w_{2}} j\right) \\
& =\left(w_{2} w_{3}\right)^{n} \sum_{i=0}^{w_{2}-1} \sum_{j=0}^{w_{3}-1}(-1)^{i+j} E_{n}\left(w_{1} y_{1}+\frac{w_{1}}{w_{2}} i+\frac{w_{1}}{w_{3}} j\right) \\
& =\left(w_{3} w_{1}\right)^{n} \sum_{i=0}^{w_{3}-1} \sum_{j=0}^{w_{1}-1}(-1)^{i+j} E_{n}\left(w_{2} y_{1}+\frac{w_{2}}{w_{3}} i+\frac{w_{2}}{w_{1}} j\right) .
\end{aligned}
$$

Letting $w_{3}=1$ in (4.23), we have the following corollary.

Corollary 4.15. Let $w_{1}, w_{2}$ be any odd positive integers. Then one has

$$
\begin{aligned}
& w_{1}^{n} \sum_{j=0}^{w_{1}-1}(-1)^{j} E_{n}\left(w_{2} y_{1}+\frac{w_{2}}{w_{1}} j\right) \\
& =w_{2}^{n} \sum_{i=0}^{w_{2}-1}(-1)^{i} E_{n}\left(w_{1} y_{1}+\frac{w_{1}}{w_{2}} i\right) \\
& =\left(w_{1} w_{2}\right)^{n} \sum_{i=0}^{w_{1}-1} \sum_{j=0}^{w_{2}-1}(-1)^{i+j} E_{n}\left(y_{1}+\frac{i}{w_{1}}+\frac{j}{w_{2}}\right) .
\end{aligned}
$$


Theorem 4.16. Let $w_{1}, w_{2}, w_{3}$ be any positive integers. Then one has the following two symmetries in $w_{1}, w_{2}, w_{3}$ :

$$
\begin{aligned}
& \sum_{k+l+m=n}\left(\begin{array}{c}
n \\
k, l, m
\end{array}\right) E_{k}\left(w_{1} y\right) E_{l}\left(w_{2} y\right) E_{m}\left(w_{3} y\right) w_{3}^{k} w_{1}^{l} w_{2}^{m} \\
& \quad=\sum_{k+l+m=n}\left(\begin{array}{c}
n \\
k, l, m
\end{array}\right) E_{k}\left(w_{1} y\right) E_{l}\left(w_{3} y\right) E_{m}\left(w_{2} y\right) w_{2}^{k} w_{1}^{l} w_{3}^{m} .
\end{aligned}
$$

Theorem 4.17. Let $w_{1}, w_{2}, w_{3}$ be any odd positive integers. Then one has the following two symmetries in $w_{1}, w_{2}, w_{3}$ :

$$
\begin{aligned}
& \sum_{k+l+m=n}\left(\begin{array}{c}
n \\
k, l, m
\end{array}\right) T_{k}\left(w_{1}-1\right) T_{l}\left(w_{2}-1\right) T_{m}\left(w_{3}-1\right) w_{3}^{k} w_{1}^{l} w_{2}^{m} \\
& \quad=\sum_{k+l+m=n}\left(\begin{array}{c}
n \\
k, l, m
\end{array}\right) T_{k}\left(w_{1}-1\right) T_{l}\left(w_{3}-1\right) T_{m}\left(w_{2}-1\right) w_{2}^{k} w_{1}^{l} w_{3}^{m} .
\end{aligned}
$$

Putting $w_{3}=1$ in (4.26) and (4.27), we get the following corollary.

Corollary 4.18. Let $w_{1}, w_{2}$ be any odd positive integers. Then one has

$$
\sum_{k=0}^{n}\left(\begin{array}{l}
n \\
k
\end{array}\right) T_{k}\left(w_{2}-1\right) T_{n-k}\left(w_{1}-1\right) w_{1}^{k}=\sum_{k=0}^{n}\left(\begin{array}{l}
n \\
k
\end{array}\right) T_{k}\left(w_{1}-1\right) T_{n-k}\left(w_{2}-1\right) w_{2}^{k} .
$$

\section{Acknowledgment}

This work was supported by National Foundation of Korea Grant funded by the Korean Government (2009-0072514).

\section{References}

[1] E. Y. Deeba and D. M. Rodriguez, "Stirling's series and Bernoulli numbers," The American Mathematical Monthly, vol. 98, no. 5, pp. 423-426, 1991.

[2] F. T. Howard, "Applications of a recurrence for the Bernoulli numbers," Journal of Number Theory, vol. 52, no. 1, pp. 157-172, 1995.

[3] T. Kim, "Symmetry $p$-adic invariant integral on $\mathbb{Z}_{p}$ for Bernoulli and Euler polynomials," Journal of Difference Equations and Applications, vol. 14, no. 12, pp. 1267-1277, 2008.

[4] T. Kim, "Symmetry identities for the twisted generalized Euler polynomials," Advanced Studies in Contemporary Mathematics, vol. 19, no. 2, pp. 151-155, 2009.

[5] T. Kim, K. H. Park, and K.-W. Hwang, "On the identities of symmetry for the $\zeta$-Euler polynomials of higher order," Advances in Difference Equations, vol. 2009, Article ID 273545, 9 pages, 2009.

[6] T. Kim, "Symmetry of power sum polynomials and multivariate fermionic $p$-adic invariant integral on $\mathbb{Z}_{p}, "$ Russian Journal of Mathematical Physics, vol. 16, no. 1, pp. 93-96, 2009.

[7] T. Kim, "On the symmetries of the q-Bernoulli polynomials," Abstract and Applied Analysis, vol. 2008, Article ID 914367, 7 pages, 2008. 
[8] T. Kim, “q-Volkenborn integration,” Russian Journal of Mathematical Physics, vol. 9, no. 3, pp. 288-299, 2002.

[9] T. Kim, “Note on the Euler q-zeta functions," Journal of Number Theory, vol. 129, no. 7, pp. 1798-1804, 2009.

[10] T. Kim, "On $p$-adic interpolating function for $q$-Euler numbers and its derivatives," Journal of Mathematical Analysis and Applications, vol. 339, no. 1, pp. 598-608, 2008.

[11] T. Kim, "On the q-extension of Euler and Genocchi numbers," Journal of Mathematical Analysis and Applications, vol. 326, no. 2, pp. 1458-1465, 2007.

[12] T. Kim, "On the analogs of Euler numbers and polynomials associated with $p$-adic $q$-integral on $\mathbb{Z}_{p}$ at $q=-1, "$ Journal of Mathematical Analysis and Applications, vol. 331, no. 2, pp. 779-792, 2007.

[13] T. Kim and Y.-H. Kim, "On the symmetric properties for the generalized twisted Bernoulli polynomials," Journal of Inequalities and Applications, vol. 2009, Article ID 164743, 8 pages, 2009.

[14] T. Kim, L.-C. Jang, Y.-H. Kim, and K.-W. Hwang, "On the identities of symmetry for the generalized Bernoulli polynomials attached to $x$ of higher order," Journal of Inequalities and Applications, vol. 2009, Article ID 640152, 7 pages, 2009.

[15] H. Ozden, I. N. Cangul, and Y. Simsek, "Multivariate interpolation functions of higher-order $q$-Euler numbers and their applications," Abstract and Applied Analysis, vol. 2008, Article ID 390857, 16 pages, 2008.

[16] H. Ozden, I. N. Cangul, and Y. Simsek, "Remarks on sum of products of $(h, q)$-twisted Euler polynomials and numbers," Journal of Inequalities and Applications, vol. 2008, Article ID 816129, 8 pages, 2008.

[17] H. Ozden and Y. Simsek, "A new extension of $q$-Euler numbers and polynomials related to their interpolation functions," Applied Mathematics Letters, vol. 21, no. 9, pp. 934-939, 2008.

[18] Y. Simsek, "Complete sum of products of $(h, q)$-extension of Euler polynomials and numbers," Journal of Difference Equations and Applications, 2009.

[19] H. J. H. Tuenter, "A symmetry of power sum polynomials and Bernoulli numbers," The American Mathematical Monthly, vol. 108, no. 3, pp. 258-261, 2001.

[20] S.-L. Yang, "An identity of symmetry for the Bernoulli polynomials," Discrete Mathematics, vol. 308, no. 4, pp. 550-554, 2008.

[21] D. S. Kim and K. H. Park, "Identities of symmetry for Bernoulli polynomials arising from quotients of Volkenborn integrals invariant under $S_{3}$," submitted. 